\title{
Optimal design of lattice metal constructions of overhead power transmission lines
}

\author{
Andrey Pustovgar ${ }^{1}$, Anton Tanasoglo ${ }^{2, *}$, Igor Garanzha $^{2}$, Liubov Shilova ${ }^{1}$, and Aleksey \\ Adamtsevich $^{1}$ \\ ${ }^{1}$ Moscow State University of Civil Engineering, Yaroslavskoye sh. 26, 129337 Moscow, Russia \\ ${ }^{2}$ Donbas National Academy of Civil Engineering and Architecture, 2 str. Derzhavin, 86123 \\ Makeyevka, Ukraine
}

\begin{abstract}
The article presents a new concept of design of power lines which allowed us to develop new unified power transmission supports, efficient and reliable ones. For the first time, a method of optimum design of overhead power lines has been developed which considered the power line as a united structural system, the relief, the atmospheric and climatic conditions, the environment pollution, the efficiency requirements, the technological and operational expenditures. The article describes the changes introduced into the requirements for the dimensions and parameters of grid metal works with the minimum mass. The optimum design, the computer-aided assembly process and the use of advanced technologies guarantee the reduction of the construction period and the increase in the maintenance period of power lines.
\end{abstract}

\section{Introduction}

The construction of overhead power lines in the Russian Federation, Ukraine, Kazakhstan and Belarus uses traditional unified support structures developed in accordance with the USSR standards [1-4]. Such support structures cannot compete with foreign ones under modern market conditions. So it is necessary to develop new unified structures for overhead power lines. The main reasons for that are following: the international norms for the determination of loads caused by climatic effects became more strict in order to make the overhead power lines more reliable; the consideration of the real work values for space support structures in the power line as a united system as well as that of supporting effects by ropes and cables is necessary [5-7].

The potential solution of the aforesaid problem lies in the improvement of power line support structures with absolute conservation of their maintenance characteristics [8-11]. Therefore, the search of a structural solution with consideration of the requirements to the analysis procedures is a very important problem as it is aimed at the conservation of the competitiveness of power lines at the world market due to the saving of metal in the process of the use of non-typical support structures.

* Corresponding author: $\underline{\text { a.v.tan} @ \text { mail.ru }}$ 
The study of the works in the field with respect to the optimum design of structures allowed us to formulate the problem which is dictated by both the modern state in the field of optimization of structures and the needs of practical construction.

The design of structures for high-voltage power lines includes the consideration of many various parameters and requirements: power line length, voltage, relief, region and electrical dimensions, land plot cost, material of structural elements, unification requirements, producer potentialities, assortment [12-16].

In the process of design of overhead power line supports, some design parameters are definitely given: the geometrical dimensions between wires, ropes and structural elements as well as the loads (wind velocity head, ice-deposits on the wires, wire break tensions). The other parameters influencing the metal grid supports vary: the support structure form (gantry support, tower support, guyed support), the span and the support height, the geometric dimensions and forms of certain structural elements (frame, beam, wire supports), the grid types, the nod joint types, the section lengths, the range of rolled metal products, the material of the structures. It is difficult to consider all these parameters within the frames of one new method even theoretically, and it is quite impossible practically. Therefore, it is expedient to solve the support optimization problem using different rational methods at different stages of the optimization process.

\section{Optimum design of grid structures}

The work [5] describes the optimum one-stage automated system for the design and production of power line structures with consideration of design-and-technology requirements of production plants. This system reduced the production time period and improved the quality of products. The system includes programming package for the strength and optimization analysis of wires, ropes and metal support structures; the fullscale modeling; the computer-aided assembly of structures. The suggested numerical optimization method allows us to optimize the geometrical dimensions and topology of power line supports, their mass, labour-intensiveness and cost simultaneously; the mass and cost parameters are by $20 \%$ lower. The well-known "TOWER" program for the analysis of overhead power line supports has been developed in the USA and recommended by the International Electrotechnical Committee. The "TOWER" program has many disadvantages in comparison with the programming package suggested by the authors: the wind loading is considered approximately, the analysis of wires and supports is performed separately; a simple sorting out of geometric parameters is performed instead of optimizing them; there is no overhead power line optimization with respect to the power line track. The authors used the developed programming package [17-19] and developed new efficient support structures with narrow bases and with one stand. The aforesaid support structures showed their good qualities in the Crimea Mountains; the portal support structures with flexible links had the mass by $30 \%$ lower than the foreign structures.

The problem of the search of optimum parameters of support structures is based on the numerical methods of minimization of non-linear target functions. The use of non-linear programming methods does not give a well defined mathematical solution of the problem, but these methods consider those necessary factors which make the problem closer to practical needs and to get an appreciable economic effect.

With consideration of the aforesaid facts, the problem of optimum design of overhead power line supports may be formulated like that: to compute the geometrical parameters of the tower with various coordinates and topology parameters for given cross-sections with minimum mass or cost and with maintenance of normative requirements and structural restrictions. The varying parameters xj may be continuous or discrete variables. 
The expert estimation of possible optimization methods shows that the most efficient methods are the following ones: the method of improved search, the Hadder-Mid method of deformed polyhedron and the direct search methods by Hooke-Jivce and Davis-SvennCampy [20-22]. A successive application of these methods allows us to get closer to the optimum variant.

The authors suggest a search method which at the initial stage allows choosing the descent direction with the determination of optimum geometric parameters. The numerical studies showed that the descent direction was determined in the process of the first iterations. For a successful search direction, a polyhedron with an "improved" vertex is formed, and the search continues until the transition step value becomes less that the given precision value. Otherwise, the search process includes a certain number of iterations. The optimum value of the target function for this method was the initial value at the transition to the next optimization method.

The suggested method of initial optimization is very efficient; it allows us to escape from the "ravines" for essential non-linear discrete problems (Fig.1) in contrast to other methods (deformed polyhedron method, Hooke-Jivce method, etc.).

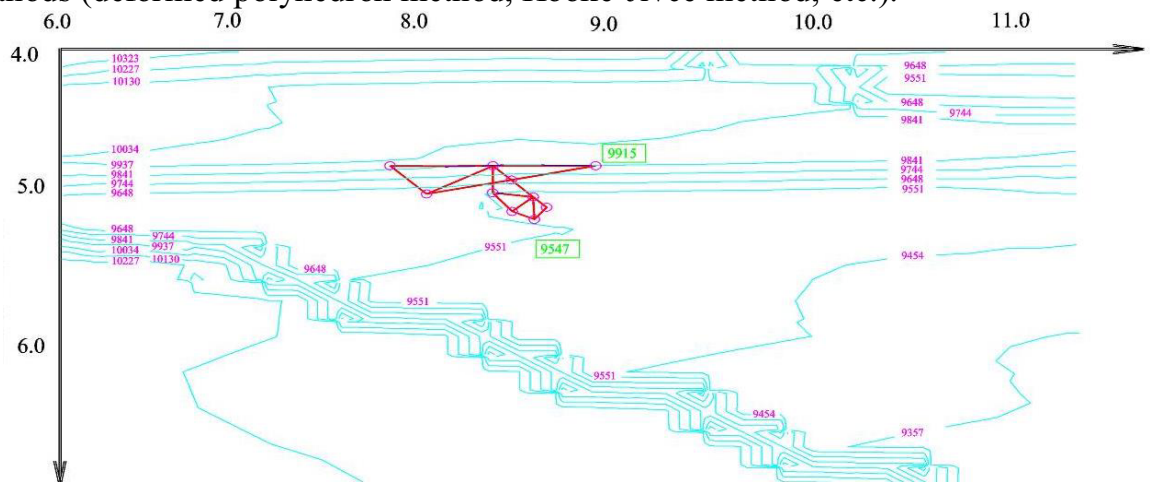

Fig. 1. Efficiency of the search through the coordinate descent method.

As the design optimum criterion, the target function is taken as the mass or the cost of the support structure. The target function is a composite non-linear function of control parameters. The discreteness and essential non-linearity of the target function sets the questions concerning the development of an algorithm providing for the convergence with the minimum mass design.

In the process of solution of the optimization problem for an overhead power line support structure, the initial data are used, the structural and other requirements are considered, the static and optimization analyses are carried out $[5,23]$.

The optimization problem is reduced to the determination of the varying parameter vector (1), which minimizes the target function and satisfies the restrictions (2) - (4) and the conditions (5), (6). The system of restrictions is formed automatically as the test for the target function, the expression of which is a difficult non-linear problem as the number of varying parameters is great $[17,24]$

$$
Z=f\left(H, L, b, Q, 1_{t}, R_{y}, K_{b}, n_{p}\right) .
$$

here $\mathrm{H}, \mathrm{h}, \mathrm{b}, \mathrm{Q}$ are the given parameters, where $\mathrm{H}$ - support height, $\mathrm{b}$-base, L - span, Q -external load vector; the values $l_{t}, R_{y}, K_{b}, n_{p}$ are the varying parameters of geometric topology, where $\mathrm{l}_{\mathrm{t}}$-panel length, $\mathrm{k}_{\mathrm{b}}$-number of bolts, $\mathrm{n}_{\mathrm{p}}$-grid type.

Write out the main restrictions for the varying parameter vector (1):

- bearing capacity of compressed and tensed structural elements, respectively: 


$$
\mathrm{N}_{\mathrm{i}} \leq \mathrm{A}_{\mathrm{i}} \cdot \mathrm{R}_{\mathrm{yi}} \cdot \varphi_{\mathrm{i}} \quad \text { and } \quad \mathrm{N}_{\mathrm{i}} \leq \mathrm{A}_{\mathrm{i}} \cdot \mathrm{R}_{\mathrm{yi}}
$$

where $\mathrm{N}_{\mathrm{i}}$-longitudinal force in the $\mathrm{i}$-th element ;

- minimum element area:

$$
\mathrm{A}_{\mathrm{i}} \geq\left[\mathrm{A}_{\mathrm{i}}\right]
$$

where $\left[\mathrm{A}_{\mathrm{i}}\right]$-minimum admissible area of the $\mathrm{i}-$ th element with respect to the ultimate flexibility or to the design requirements;

$$
\mathrm{J}_{\mathrm{i}} \geq \tilde{\mathrm{O}}_{\mathrm{i}} \geq \mathrm{j}_{\mathrm{i}},
$$

where $\mathrm{J}_{\mathrm{i}}$ and $\mathrm{j}_{\mathrm{i}}$-upper and lower limits for the varying parameter range.

The main conditions which the varying parameter vector meets (1):

- equilibrium condition:

$$
\mathrm{k}(\mathrm{x}) \cdot \mathrm{B}-\mathrm{P}(\mathrm{x})=0 \text {, }
$$

where $\mathrm{k}(\mathrm{x})$-system rigidity matrix;

$\mathrm{B}$ - unit translation vector;

$\mathrm{P}(\mathrm{x})$ - external loading vector;

- deformation compatibility condition:

$$
\Delta \mathrm{L}_{\mathrm{Ni}}\left(\mathrm{N}_{\mathrm{i}}, \mathrm{A}_{\mathrm{i}}, 1_{\mathrm{i}}\right)=\Delta_{\mathrm{i}}(\Delta \mathrm{x}, \Delta \mathrm{y}, \Delta \mathrm{z})
$$

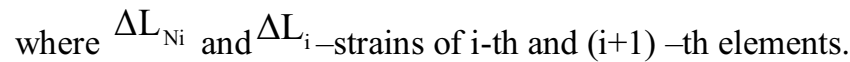

The geometric topology of the power line support is given with consideration of the restrictions (2) - (4) for the variables. These restrictions are directed at the conservation of a certain system form and the correspondence of varying parameters to the design requirements [25-27].

The optimum design algorithm for overhead power line supports is implemented in the "USL" programming package (Fig.2). 


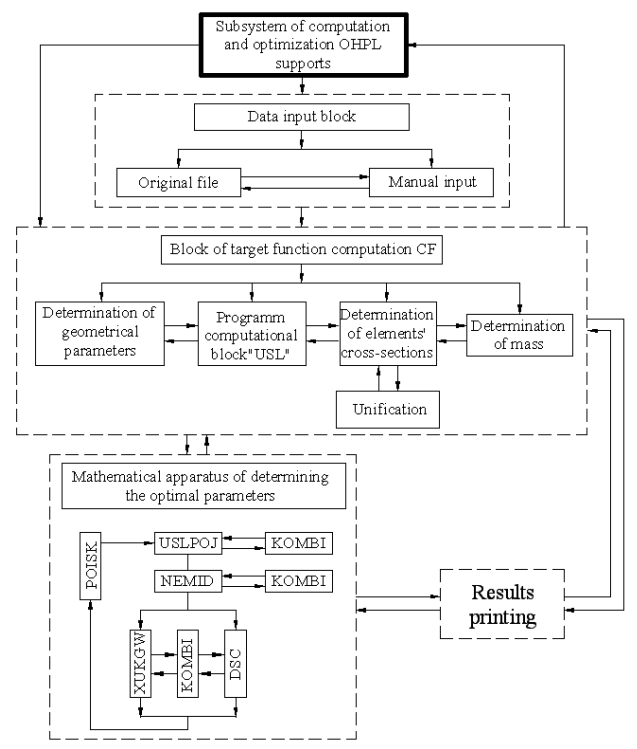

Fig. 2. Principle block diagram of the "USL" program.

The optimization problem has been solved through the numerical methods of nonlinear programming which do not use the derivatives. The basic idea of the search is the use of preceding information for the construction of improved solution with the help of iterative procedures. The research work done allowed us to recommend the following methodology of the use of various mathematical methods and their succession. At the first stage, the optimum grid type for given geometric dimensions is determined (for self-supporting supports) with the use of the developed combinatorial algorithm. The value of the target function for the optimum grid type becomes the initial value for the following iterative computations.

Then a regular polyhedron is set which has a form of a regular simplex. The descent direction is determined. For new varying parameter values, the optimum grid type is chosen through the combinatorial algorithm (KOMBI), and the target function value is computed.

Further, a polyhedron is used for the determination of the optimum; one of the vortex of the structure was the optimum one at the precedent stage (USLPOY). The search continues through the deformed polyhedron method (NEMID). Then the optimum grid type is determined through the combinatorial algorithm, and the geometric dimensions of the support are obtained as well.

At the last stage, the efficiency function is optimized through one of the coordinate descent methods. Here the most efficient method is the direct search method by HookeJives (XUKGV) as it gives the "best" (minimum) value of the target function. The iterative search process is completed by the choice of the optimum grid type through the combinatorial algorithm.

The initial design data with the constant values come from the main program (or introduced through the key-board) into the optimization "POISK" program. The target function minimum for the minimum mass structure may be obtained through one of optimization method or by a combination of these methods (Fig.2). the sub-program "CF" for the computation of the target function includes several computation blocks which determine different parameters for each element group: belts, braces, strut frames, diaphragms.

The analysis of optimization results (presented in Table 1) proves the following conclusions: there exists an objective value of the structure mass for the same load values 
and support dimensions; this mass value may be achieved from different initial design variants with different grid types, diaphragms, nod joints, cross-sections. The grid topology does not influence the end results.

Table 1. Optimization results for the "Y220-2+5" support with different initial states.

\begin{tabular}{|c|c|c|c|}
\hline \multicolumn{2}{|c|}{ Initial support } & \multicolumn{2}{c|}{ Optimal support } \\
\hline $\begin{array}{c}\text { Geometric } \\
\text { tomolnov }\end{array}$ & Diaphragm type & Geometric topology & Diaphragm type \\
\hline & & $1-1,2-2,3-3$ & \\
\hline
\end{tabular}

The program may get the information concerning every step of the iterative process of the problem solution for the purpose of its analysis and the correction of some parameters. A graphic representation of the computation process is provided. The optimization results are obtained in the form of tables.

The programming package may be used for the solution of optimum design problems for all types of overhead power line supports; besides, it is possible to add some computation blocks for the solution of other optimization design problems. The optimization computations for various support types for overhead power lines considerably improve the initial design variants. This fact proves the expediency of the aforesaid approach to the support optimization, as this approach gives a rational structure form which meets the optimum criterion. The research works done considered a power line as a united structural system; they took into account the space work of the support structures as well as their technological and economic efficiency.

\section{Development of new optimum support structures for overhead power lines with $110 \mathrm{kV}$ voltage}

The optimization computation for tower supports (overhead power lines with $110 \mathrm{kV}$ voltage) was carried out through the programming package "USL" [28].

In the process of optimization of anchor support structures, the given parameters were the base and the width of the upper part of the shaft which is determined by the electrical dimensions. The varying parameters were the length and the number of panels, the length of belt sections, the grid type and the shaft diaphragm type, the type of nod mating of struts and belts.

For the development of new supports the following conditions are taken:

-Support material: low-carbon steel C245 (Ry= $240 \mathrm{MP})$; 
-Current-carrying wires (steel-and-aluminum; grade AC 240/32);

-Overhead ground-wire cable C50;

-Wind span lwind=ldim ; weight span [29];

-The climatic conditional are taken in accordance with Chapter 2.5 [30].

The geometric forms of developed optimum anchor supports (overhead power lines with $110 \mathrm{kV}$ voltage) are presented in Fig.3. The comparison of mass values for typical and optimum supports is given in Table 2.
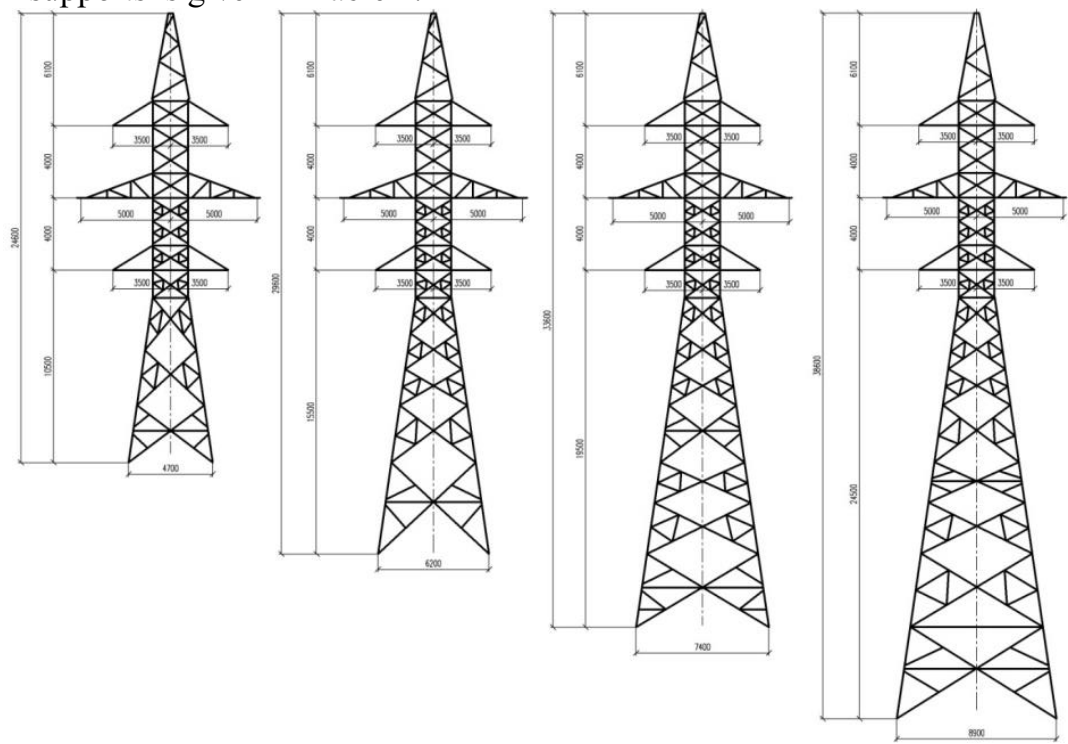

Fig. 3. Geometric forms of optimum anchor supports "Y110-2": a) Y110-2 type support; b) Y1102+5 type support; c) Y110-2+9 type support; d) Y110-2+14 type support.

The optimization of the "Y110-2+9" support resulted in the mass reduction from 11.391 $\mathrm{t}$ to $8.383 \mathrm{t}$ (by $35.9 \%$ )/ the belt angle in the structure of the $9 \mathrm{~m}$ high stand for the optimum support was replaced by another belt angle grade ( L 180x11 was replaced by L160x10).

Table 2. Comparison of typical "Y110-2" supports with the optimum ones.

\begin{tabular}{|c|c|c|c|c|c|}
\hline \multirow{2}{*}{ Support type } & \multicolumn{2}{|c|}{ Support mass* } & \multicolumn{2}{c|}{ Saving of material for 1 support } \\
\cline { 3 - 6 } & $\begin{array}{c}\text { Typical support, } \\
\text { KM }\end{array}$ & $\begin{array}{c}\text { Optimal support } \\
\text { KMD }\end{array}$ & kg & $\%$ \\
\hline 1 & Y110-2 & $\frac{7704}{8002}$ & $\frac{5676}{5910}$ & $\frac{2028}{2092}$ & $\frac{35.7}{35.4}$ \\
\hline 2 & Y110-2+5 & $\frac{9717}{10095}$ & $\frac{7185}{7481}$ & $\frac{2532}{2614}$ & $\frac{35.2}{34.9}$ \\
\hline 3 & Y110-2+9 & $\frac{11391}{11834}$ & $\frac{8383}{8728}$ & $\frac{3008}{3106}$ & $\frac{35.9}{35.6}$ \\
\hline 4 & Y110-2+14 & $\frac{14643}{15212}$ & $\frac{10476}{10908}$ & $\frac{4176}{4304}$ & $\frac{39.8}{39.5}$ \\
\hline
\end{tabular}

*the numerator shows the mass of support without painting; the denominator shows the mass of support with zinc coating. 
The results of the research work are introduced into the process of production, erection and maintenance of the "Y110-2+14" optimum support (overhead power line "Kremenching-GPP4"; voltage: $110 \mathrm{kV}$ ) at the territory of Poltava mining integrated works, Komsomolsk, Ukraine (Fig.4). The efficiency of the aforesaid support is the mass reduction by $39.5 \%$ in comparison with the typical support due to a more precise determination of forces in the space model of the support, the re-computation of the buckling coefficient, the introduction of a composite strut frame grid, the choice of the optimum profile type and the nod mating.

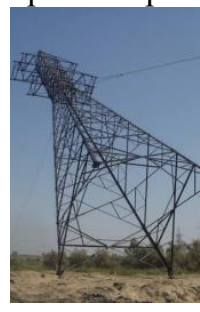

a)

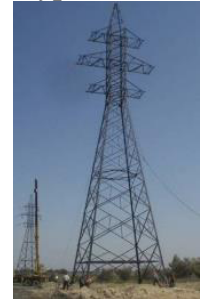

b)

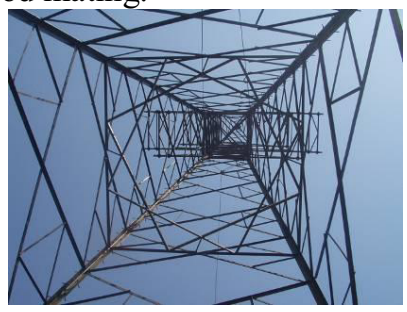

c)

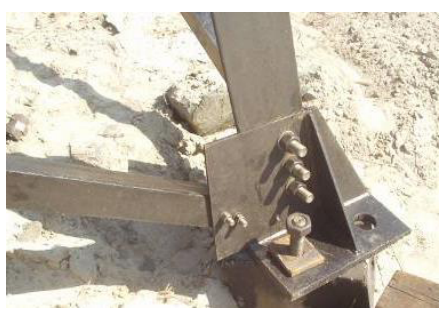

d)

Fig. 4. The optimum two-chain anchor support "Y110-2":The support is being erected; b) The general view of the support; c) The composite strut frame grid of the shaft; d) The nod of fixation of the support on the foundation (shoe).

\section{Conclusions}

The authors developed a methodology of optimization of parameters of the geometric form and type of the lattice for the structures of overhead power line supports which meets the requirements of construction norms and rules.

The search of minimum target function is performed through a combination of suggested and existing methods of non-linear programming.

The developed method of optimum design of overhead power line supports is universal, and it may be applied to other structures - the supports for wind power units and the overhead supports for radio-relay communication.

The developed system of one-stage automated design of power line structures includes a programming package for optimization computations of wires, ropes, support structures and foundation structures.

The programming package was successfully used for the development of two-chain optimum anchor supports for overhead power lines with $110 \mathrm{kV}$ voltage which are technological with respect to their production and erection and meet the Ukraine climatic conditions. The mass of optimum supports is by $35.2 \%$ to $39.8 \%$ less as compared with typical "VL $110 \mathrm{kW"} \mathrm{supports.}$

\section{References}

1. K.P. Kriukov, B.P. Novgorodtsev, Constructions and mechanical analysis of power transmission line (1979)

2. D. Vinogradov, Construction of overhead power transmission lines 35-500 $\mathrm{kV}$ with severe track (1983)

3. Ye. Gorokhov, S. Shapovalov, Ye. Udod, et al. Growth of reliability and life of the power supply network construction (1997)

4. K. Kryukov, The crossings of transmission lines through of large water areas (1982)

5. E.V. Shevchenko, Refinement of steel constructions of power transmission line support (1999) 
6. IEC 60826. Design criteria of overhead transmission lines (2003)

7. EN 50341-1: Overhead electrical lines exceeding AC $45 \mathrm{kV}$. Part 1: General requirements - Common specifications (2009)

8. Ya. Nazim, A. Tanasoglo, Met. Const., 49-61 (2015)

9. Probabilistic design of overhead transmission lines: Companion document to "Improved design criteria of overhead transmission lines based on reliability concepts" (2000)

10. B.C. Wadell, Transmission Line Design (Artech house, Norwood, 2005)

11. H. Li, H. Bai, Prog. in Nat. Sc., 899-911 (2006).

12. Ye. Gorokhov, M. Kazakevich, et al., Aerodynamics of the power supply structures (2000)

13. G. Diana, S. Bruni, F. Cheli, F. Fossati, A. Manenti, EACWE, 1759-1766 (1997)

14. M. Gaudry, F. Chore, C. Hardy, E. Ghannoum, Increasing the ampacity of overhead lines using homogeneous compact conductors (2011)

15. Y. Chen, M. Farzaneh, E. Lozowski, K. Szilder, IWAIS, 1-8 (2000)

16. A.G. Sokolov, Support of power transmission lines (calculation and construction) (1961)

17. Ye. Shevchenko, V. Glukhov, A. Tanasoglo, Metal Structures, 31-39 (2010)

18. Ya. Nazim, Ye. Shevchenko, Mod. Ind. and civ. Const., 83-95 (2014).

19. Ye. Shevchenko, Ya. Nazim, A. Tanasoglo, I. Garanzha, Proc. Eng., 1033-1040 (2015).

20. P. Couneson, J. Lamsoul, D. Delplanque, Proc. CIGRE, 18-76 (2013)

21. G. Arzhakov, Metal structures. Special buildings and structures (2002)

22. A. Perelmuter, Computational models of structures and the possibility of their analysis (2002)

23. A. Tanasoglo, I. Garanzha, V. Glukhov, Met. Struct., 39-48 (2015)

24. J. Lamsoul, J. Rogier, P. Couneson, A. Van Overmeere, Belgian experience on initiatives to improve the capability of existing overhead lines (2000)

25. DBN B.2.6 - 163:2010 Steel structures. Standards of design, manufacturing and installation (2011)

26. Manual for design of steel structures of overhead transmission line supports (OHTL) and power outdoor switchgear (OSG) substations with voltage up to $1 \mathrm{kV}$ (to SNiP II23-81*) (1989)

27. Design of Latticed Steel Transmission Structures, American Society of Civil Engineers. ANSI/ASCE 10-90, A.N.S.I. (1991)

28. A. Tanasoglo, Mod. Ind. and civ. Const., 5-14 (2015)

29. M. Gaudry, F. Chore, C. Hardy, Proc. CIGRE, 180-201 (2008)

30. Rules for electrical installation. Head 2.5 «The overhead power transmission lines voltages above $1 \mathrm{kV}$ to $750 \mathrm{kV}$ 》 (2006) 